\title{
THE PREDICTION GENERATOR: A TOOL FOR CREATING MEANINGFUL SPECULATIONS
}

\author{
José De La O CAMPOS ${ }^{1,3}$, David GUEMES-CASTORENA ${ }^{2,3}$ \\ ${ }^{1}$ School of Architecture, Art and Design, Tecnologico de Monterrey, Mexico \\ ${ }^{2}$ School of Engineering and Sciences, Tecnologico de Monterrey, Mexico \\ ${ }^{3}$ Tecnologico de Monterrey, Writing Lab, TecLabs, Vicerrectoría de Investigación y \\ Transferencia de Tecnología, Monterrey 64849, NL, Mexico
}

\begin{abstract}
As Industrial Design is a practice that uses prospective thinking, the quality of these ideas, translated into future scenarios, speculations, or futuristic design concepts, must be innovative enough to trigger discussion and provoke, but at the same time, believable and close to an area of what could happen. It might be difficult for industrial design students and professionals to understand the balance between what is possible and fantasy.

This paper describes an ideation tool named The Prediction Generator $\odot$, an ideation instrument developed by Studio José de la $\mathrm{O}$ designed to help designers, researchers, future thinkers, and in this case, design students to generate, validate, and articulate any design brief, research hypothesis or future Scenario. The Prediction Generator $\odot$ is a canvas-like toolkit that works by filling six consecutive slots: Desires, Similar Cases, Patterns, Limitations, Questions and Hypothesis. Later, this paper describes different examples of how this tool was deployed on academic projects, ranging from speculative design projects to real-life social design assignments, and the perception of the students before and after they used this tool and a description on how this tool was applied.
\end{abstract}

Keywords: Industrial design, innovation \& creativity, toolkit, speculative design, critical thinking, educational innovation

\section{INTRODUCTION}

Design is a practice that heavily relies on prospective thinking. As design operates in uncertainty and it gives form or visibility to new futures that are constantly changing[1], it is crucial that any designer, whatever the discipline is, from speculative or critical design or more commercial-driven practice, to develop a future-thinking mindset. One might think that a traditional designer must design for today's needs, but, as some design projects could take several years to completion, a designer must construct for the problems of tomorrow. One common problem that could happen during a medium to long term design projects is that by the time the project becomes a reality, the information and criteria used to build the can become outdated, therefore, rendering the result of this project useless. Using anticipatory thinking in the conception of a design concept could be the difference that makes a project successful. This paper describes The Prediction Generator, an ideation tool that initially was designed at delaO Design Studio in Mexico City, a design research studio that utilizes critical and speculative design as part of its main practice. The need to have a tool like this started as some of its less experienced designers in the team were having trouble generating believable speculative concepts, or as we call them in this paper, Believable Pararealities. After analysing how these unexperienced designers came up with these ideas, it was identified that crucial factors were somehow ignored. These factors ranged from the lack of understanding of the context where these speculations were supposedly happening, ignoring or neglecting the limitations that could influence the possibilities that those ideas might or might not happen, and a lack of benchmark research that could help to shape the foundations of an original idea. A canvas-like toolkit was conceived, inspired on the Business Model Canvas, a toolkit that segments different aspects of a business model and allows the user to prototype business ideas with a more visual thinking approach [2]. It was identified that for generating these speculative ideas, one could explore different conceptual approaches, go back and forth from each segment, and it would be not difficult if one needs to change or do a U-turn in the research process. After some prototyping of the tool itself and 
some successful testing, The Prediction Generator was registered under a Creative Commons license and published through the studio website where it is available for free since 2018. In addition to a full description of the toolkit, this paper will describe what we mean by Believable Pararealities.

The remaining part of the paper proceeds as follows: first, we will introduce the concept of believable pararealities; secondly, we will explain every segment of The Prediction Generator and how it has helped us in teaching prospective thinking in design, concept development, and briefing generation. Lastly, we will conclude with some insights on the use of this toolkit as a purposeful resource.

\section{BELIEVABLE PARAREALITIES}

As mentioned before, prospective thinking and speculation linger in dangerous areas where these ideas, if not crafted correctly, they could be dismissed by the audience it tries to relate to. To create these believable near realities, we would need to borrow some tactics from fiction and craft what we call "Pararealities." Let us explore the idea behind a "Parareality." We use the prefix "para" as etymologically brings a meaning of "besides," "next to," "near," "from," "against," or "contrary to." We like to explore the different ideas of having a Reality that exists, using all those prepositions, together with what we call "Our Reality." We find inspiration on Floyd Merrell, as he used this word on the title of his seminal book that explores among a broad epistemological view, how to process and create fiction. Merrell finds a complex psychological, linguistic, and social negotiation between a perceived world and a real-world [3]. Awazuhara elaborates a "Para-reality" (with a hyphen) as metaphoric images ranging from myths to Anime, which could be described as imaginary constructs that proposes a model that portrays a world, which comes as a selective abstraction of a "real world" [4]. Merrell argues that the fictional idea must be accepted as a self-evident, supported by subsequent arguments, proofs, or actions [5]. The premise or proposal of the concept must be believable. These axiomatic, but fictional ideas, when presented narratively, must also exist with a negation from the spectator in the sense that the spectator understands that these ideas are outside reality. However, what happens when we can go beyond Fiction from a narrative, and we would like to turn them into what Dunne \& Raby call "Functional Fictions?" A "Functional Fiction" is an imaginary but believable conceptual starting point that describes an everyday situation that could help to explore possible consequences [6]. This starting point can be, for example, a design brief that could challenge the way we can think about one specific emerging technology and the impact it might cause in society: a "Parareality" in the shape of a design brief. By going beyond a narrative approach into a more functional approach can be extremely useful for the design discipline as it continually deals with uncertainty. There is an increasing demand for understanding what is next, as the rate of change accelerates, and the future grows more uncertain, not only by designers but for other decision-making stakeholders to approach science fiction for strategic purposes [7]. A popular design tactic is design fiction, that brings together approaches of product design and science fiction, bringing prototyping into narrative to come up with what is called a "diegetic prototype," a sort of movie prop that intents to, as Bruce Sterling suggests, "to suspend disbelief about change" [8]. Design Fiction is a handy tactic as it helps us to materialize Pararealities and to help us to see how the world could be [9]. To achieve these believable Pararealities, Auger suggests building "perceptual bridges" that could connect the concept with the audience. Even if he elaborates on a number of these bridging techniques, but for the sake of this paper, we will only mention three.

- Design for context: Where the speculation exists? A specific space like the home, office, or cultural or political situation based on current development or trends.

- $\quad$ The uncanny: Desirable discomfort. Utilizing the right amount of provocation, to look for a balance between invoking a sense of familiarity while at the same time being foreign.

- Verisimilitude: Design fiction or design faction: Crafting a complex narrative using the real-life context where the fictitious concept is to be applied and taking advantage of contemporary media, familiar settings, and complex human desires and fears [9].

In conclusion, to create believable "Pararealities," the designer must be aware that the premise should be self-evident by being coherent with the real world, crafting each element from the bit and pieces of existing phenomena, and understanding the limitations where these Pararealities occur. Later in this paper, we will explain how our toolkit can be used to generate these believable Pararealities. 


\section{THE PREDICTION GENERATOR}

The Prediction Generator is an ideation instrument developed by delaO Design Studio aimed to help designers, researchers, and future-thinkers to generate, validate, and articulate a Parareality. Later we describe how it was used to create design briefs, research hypotheses. This tool is divided into six sequential parts or "slots" that starts with an idea and continuously validate it through each slot. To illustrate how the toolkit works, we will use an example of a concept development done for the Dutch design magazine Frame.

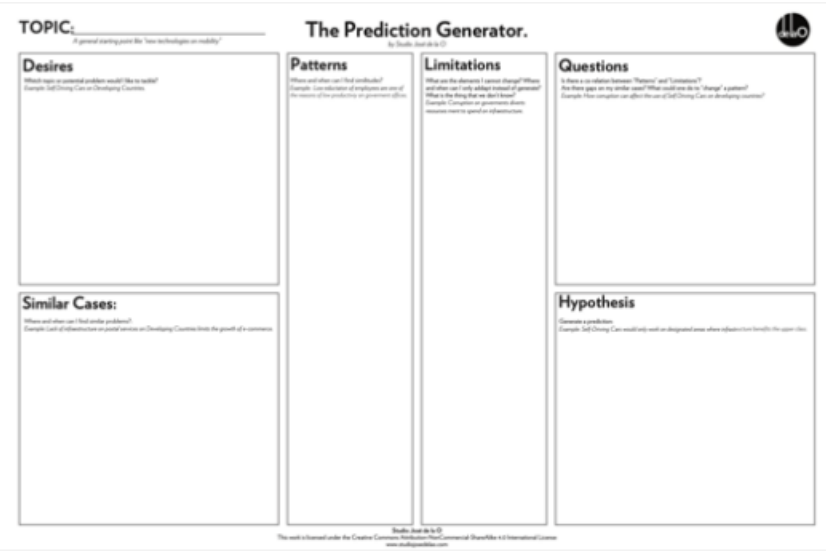

Figure 1. The Prediction Generator

\subsection{Slot 1: Desires}

The first slot is the starting point of the Prediction Generator methodology, where the user starts with an idea. This first slot motivates the designer to start with their intuition and reflect on its preferences; this is the first stage where a Parareality takes shape. As we will review later, this toolkit functions as a methodology to articulate the original idea prospectively, and to validate the ideas' depth and meaningfulness.

To start, one should write as simple as possible, in a sentence form, a description of what one would want to explore. This sentence should try to answer a "what?" and "where?" questions. Examples could be "self-driving cars in developing countries" or "virtual retail spaces in the near future." It is essential to state in the sentence an element of context, so the idea does not get too open. In contrast, we discourage not to propose a prediction at this stage, as one advances through each slot, one could start catching a glimpse of possible solutions or hypothesis on these ideas. Even if it can be possible to do so, we think that this could generate biases and will make it more challenging to come up with a meaningful result. In other words, one this sentence is answering the What and Where questions, but not explaining How. To illustrate this, we will use as an example a project developed for Dutch design magazine Frame, to come up for a concept of the Future of Retail for their "Design Challenge" column [10]. We choose the sentence "virtual retail spaces in the near future" as our starting statement.

\subsection{Slot 2: Similar Cases}

The second step on this instrument, called Similar Cases, encourages the user to come up with a list of analogue examples based on the idea written on the "Desires" slot. This step functions in one hand to develop benchmark research and in the other to start validating the depth of this idea. It is encouraged to be creative in the way the user finds these similar cases, as this list will provide the foundations for the final hypothesis. Another way of understanding this slot is where one brings all the real-life ecology where the Parareality will happen.

To illustrate this, let us go back to the Design Frame Challenge example. To find similar cases, one should mention traditional retail spaces like malls or department stores, but also, one could enlist things like "Play Station Console." It may feel strange in the beginning to mention a gaming console when we are exploring retail space, but if we do a bit of research on this platform, we will find out that inside the PlayStation virtual space, one can find an online store, apps by other companies and the actual games, where sometimes, there are online-purchase experiences. In a way, the PlayStation platform functions as a virtual retail space if we think about it. This last example can bring a bit of the research that can go behind these parts of the methodology. 


\subsection{Slot 3: Patterns}

On the third step, one must analyse all the similarities, commonalities, and repetitions of similar cases and list as many as possible. By creating this list, the user of this tool starts to gather information on how this idea could be developed. To return to our Frame Challenge example, we questioned the commonalities between the Play Station 4 platform and other physical retail places, like a shopping mall. We figured that on both, there are several space-with-in-the-space where one navigates through them and having different experiences, most of them controlled by a brand. In the case of a shopping mall, every mall has a defined brand experience, and each store is separated in terms of brand experience. Similarly, the virtual space of the PlayStation4 works on its apps and games, where each game is an entirely immersive experience from the central platform. On both the mall and the PlayStation, the path taken to go to the final experience does not compete for the attention of the user, giving enough ques to take a fast direction to the desired experience that one could make purchases. In this example, we use our intuition to create the analogy as this may not be as apparent as other people outside this exercise. When the users of this tool find themselves with much content, it can be interpreting that the idea has potential. In contrast, when one finds lots of friction on finding these commonalities, it is recommended instead to do more research or reframe the Desires slot.

\subsection{Slot 4: Limitations}

On this step, one must reflect on the context limitation of one's idea. In the toolkit, it is encouraged to make a list of the elements that cannot be changed and the situations that one can only adapt rather than create, being thoughtful about the things that one does not know. This step is crucial to produce meaningful speculation as most of the time, designers and future thinkers tend to ignore certain aspects of the context one is speculating upon. This slot also helps the designer to reflect on the meaning where this speculation is taking place, and to produce believable speculation, acknowledging and listing the limitations is a good start. Are these limitations cultural? Technological? Or only physically not possible? These questions help the designer to analyse the context where the Parareality happens, and it helps to eliminate all the factors or ideas that could not be relatable to the audience. Going back to our example, some of the limitations found are the fact that on a virtual space, all the objects found are virtual. So how could one go shopping in a virtual word? In contrast, one of the limitations of a physical retail space is the fact that it takes a lot of time and resources to change the design of the area. That is one of the reasons why most of the department stores rely on their storefront to get the attention of shoppers. A storefront is more accessible to change and refurbish rather than the entire shop. By analysing the limitations of our similar cases in the context of our main idea, we can get more realistic speculation, therefore, more relatable to the audience intended.

\subsection{Slot 5: Questions}

This slot encourages the Designer to use questions to look back into all the previous information generated in the toolkit. These questions are created by identifying correlations between the "patterns" and "limitations" slots, identifying any gaps among similar cases, or simply what must be done to change a pattern. The reason to frame this into a question is that we think that by asking a question, instead of going straight into speculation, one can process the idea better and open different possibilities. On the first versions of this tool, it was expected to jump straight from the limitations to the hypothesis. After testing this tool, it was decided that an extra step had to be used to process better all the information. By asking a question, it helps to create the foundations of the hypothesis.

We are going back to our recurrent example, the virtual retail space of the future. At this stage, we can see that the idea is taking shape: a virtual space inspired by a gaming console that could bring a similar experience as a shopping mall or a department store. The limitation of the virtual space is that one cannot bring digital objects to reality. So, a question could take the shape of something like, "What would we need to bring real objects from the digital space?" "How these objects would look like with an advanced 3D printer?", "How a shopper could buy a product seamlessly in a virtual world?" or "What would it be the financial consequences of shopping in a virtual world?"

This reflection process helps not only to revisit the previous information, but it can start giving the shape of the final Hypothesis. 


\subsection{Slot 6: Hypothesis}

The last step is to come up with a prediction. One must try to answer two or more of the questions posed during the previous stage with one solid logic affirmation and write it down. One can write several of these affirmations to describe the final hypothesis. In our example, we could write it as follows: "In the near future, retail space will be virtual. As in a virtual world, laws of physics do not exist, and space could not have the physical constraints of the real world. All the objects that exist in the digital space will be able to 3D print them. A 3D printer will be closer to a gaming console rather than an electrical appliance. The "shopper" would "purchase" these virtual products just by virtually grabbing them, on a seamless transaction. The objects will be printed instantly while the "shopper" is still on the virtual experience."

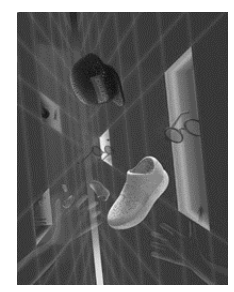

Figure 2: Frame Design Challenge

\section{CONCLUSIONS}

The first time the Prediction Generator was released was at José de la O's workshop during the 2017 Primer Conference in San Francisco. Since then, we have been using the Prediction Generator to teach students to come up with meaningful speculations, and as a tool to validate design research and to create design briefs. These projects range from product, to social, and to speculative design, as the Critical Data design project where students were encouraged to develop artifacts by stressing critical thinking in their design process [11]. These examples suggest the essential role for The Prediction Generator in teaching prospective thinking to design-students and junior designers. We encourage the academic design community to experiment further and validate tools such as this one, A reasonable approach to tackle this issue could be as the one suggested by Duran-Novoa et al. [12] who quantify the relevance of these methodologies.

\section{REFERENCES}

[1] Engeler, B. (2017). Towards prospective design. The Design Journal, 20(sup1), S4591-S4599. https://doi.org/10.1080/14606925.2017.1352956

[2] Johnson, E. A. J. (2012). Business Model Generation: A Handbook for Visionaries, Game Changers, and Challengers by Alexander Osterwalder and Yves Pigneur. Hoboken, NJ: John Wiley \& Sons, 2010. 281 + iv pages. US\$34.95. Journal of Product Innovation Management. https://doi.org/10.1111/j.1540-5885.2012.00977_2.x

[3] Taylor, V. E. (2000). Para/inquiry postmodern religion and culture. London: Routledge.

[4] Awazuhara, A. (2004). Beauty never dies: images of para-reality in Japan. Carpinteria, CA: Pacifica Graduate Institute.

[5] Merrell, F. (1983). Pararealities: the nature of our fictions and how we know them. Amsterdam: Benjamins.

[6] Dunne, A., \& Raby, F. (2013). Speculative everything: Design, fiction, and social dreaming. Speculative Everything: Design, Fiction, and Social Dreaming. https://doi.org/10.1093/jdh/epv001

[7] Zaidi, L. (2019). Worldbuilding in Science Fiction, Foresight and Design. Journal of Future Studies, 23(June 2019), 3-14. https://doi.org/10.6531/JFS.2019

[8] Bosch, T. (2012, March 2). Sci-Fi Writer Bruce Sterling Explains the Intriguing New Concept of Design Fiction. Retrieved from https://bit.ly/3cMWgN4 [9] Malpass, M. (2019). Critical design in context: history, theory, and practices. London: Bloomsbury Academic, an imprint of Bloomsbury Publishing Plc.

[9] Auger, J. (2012, September 12). (PDF) Why Robot? Speculative design, the domestication of ... Retrieved February 29, 2020, from https://www.researchgate.net/publication/323639975_Why_Robot_Speculative_design_the_dom 
estication_of_technology_and_the_considered_future

[10] Buy Now. (2018, July). Frame, (123), 34-35.

[11] de la O Campos, J. R., \& Güemes-Castorena, D. (2019). Critical data: Teaching design through critical design, physical computing, and digital data. Proceedings of the 21st International Conference on Engineering and Product Design Education: Towards a New Innovation Landscape, E and PDE 2019, (September). https://doi.org/10.35199/epde2019.26

[12] Duran-Novoa, R., Lozoya-Santos, J., Ramírez-Mendoza, R., Torres-Benoni, F., \& VargasMartínez, A. (2019). Influence of the method used in the generation of valid engineering concepts. International Journal on Interactive Design and Manufacturing, 13(3), 1073-1088. https://doi.org/10.1007/s12008-019-00577-4 